\title{
Dispersion equations for field-aligned cyclotron waves in axisymmetric magnetospheric plasmas
}

\author{
N. I. Grishanov ${ }^{1}$, M. A. Raupp ${ }^{1}$, A. F. D. Loula ${ }^{1}$, and J. Pereira Neto ${ }^{2}$ \\ ${ }^{1}$ Laboratório Nacional de Computação Científica, Av. Getulio Vargas, 333, Quitandinha, 25651-075, Petrópolis, RJ, Brasil \\ ${ }^{2}$ Universidade do Estado do Rio de Janeiro, UERJ, Av. São F. Xavier, 538, Maracana, 20550-013, Rio de Janeiro, RJ, Brasil
}

Received: 13 October 2005 - Accepted: 20 December 2005 - Published: 23 March 2006

\begin{abstract}
In this paper, we derive the dispersion equations for field-aligned cyclotron waves in two-dimensional (2-D) magnetospheric plasmas with anisotropic temperature. Two magnetic field configurations are considered with dipole and circular magnetic field lines. The main contribution of the trapped particles to the transverse dielectric permittivity is estimated by solving the linearized Vlasov equation for their perturbed distribution functions, accounting for the cyclotron and bounce resonances, neglecting the drift effects, and assuming the weak connection of the left-hand and right-hand polarized waves. Both the bi-Maxwellian and bi-Lorentzian distribution functions are considered to model the ring current ions and electrons in the dipole magnetosphere. A numerical code has been developed to analyze the dispersion characteristics of electromagnetic ion-cyclotron waves in an electron-proton magnetospheric plasma with circular magnetic field lines, assuming that the steady-state distribution function of the energetic protons is bi-Maxwellian. As in the uniform magnetic field case, the growth rate of the proton-cyclotron instability (PCI) in the 2-D magnetospheric plasmas is defined by the contribution of the energetic ions/protons to the imaginary part of the transverse permittivity elements. We demonstrate that the PCI growth rate in the 2-D axisymmetric plasmasphere can be significantly smaller than that for the straight magnetic field case with the same macroscopic bulk parameters.
\end{abstract}

Keywords. Magnetospheric physics (Energetic particles, trapped) - Space plasma physics (Kinetic and MHD theory; Waves and instabilities)

\section{Introduction}

Cyclotron waves are an important constituent of plasmas in solar corona, solar wind and planetary magnetospheres. As is well-known, the energetic particles (electrons, protons, heavy ions) with anisotropic temperature (pressure) can excite a wide class of cyclotron wave instabilities. Ki-

Correspondence to: N. I. Grishanov

(nikolay@1ncc.br) netic theory of electromagnetic cyclotron waves/instabilities in the straight magnetic field plasma is well developed (see, e.g. the publications by Sagdeev and Shafranov, 1961; Cornwall, 1965; Kennel and Petschek, 1966; Horng, 1977; Cuperman, 1981; Gomberoff and Neira, 1983; Kozyra et al., 1984; Bespalov and Trakhtengerts, 1986; Bittencourt, 1986; Gary, 1993; Xue et al., 1996; Dasso et al., 2002; Khazanov et al., 2003; and bibliography therein). However, the models of uniform plasmas confined in the straight magnetic field are suitable to study the wave processes in the solar wind and quite rough for the planetary magnetospheres, which are three-dimensional in the general case. As a more suitable approximation, the internal part of the Earth's magnetosphere, including the region of the Earth's radiation belts and the geostationary orbit, can be considered as a twodimensional (2-D) axisymmetric plasma configuration with point dipole magnetic field lines. Another interesting 2-D magnetosphere-like plasma model is a configuration with circular magnetic field lines (Grishanov et al., 1998), which is artificial but mathematically simpler and helpful to describe the principal wave processes in the Earth's magnetosphere.

The main feature of 2-D magnetospheric plasmas is the fact that i) the parallel velocity of charged particles moving along the geomagnetic field lines is not constant (in contrast to a straight uniform magnetic field case), and ii) the ambient geomagnetic field is axisymmetric and has one minimum in the equatorial plane. As a result, all plasmaspheric particles are magnetically trapped, bouncing between the mirror points (where their parallel velocity is equal to zero), and the wave-particle resonance conditions should take into account the cyclotron and bounce resonances. Accordingly, the instabilities of the cyclotron waves in the Earth's magnetosphere/plasmasphere should be analyzed by solving Maxwell's equations with a correct "kinetic" dielectric tensor, which can be obtained by solving either the Vlasov or the drift-kinetic equation for trapped particles, taking into account a 2-D nonuniformity of the geomagnetic field and plasma parameters. The drift-kinetic equation is suitable to study the wave properties in collisionless magnetized plasmas in a frequency range much less than the ion-

Published by Copernicus GmbH on behalf of the European Geosciences Union. 


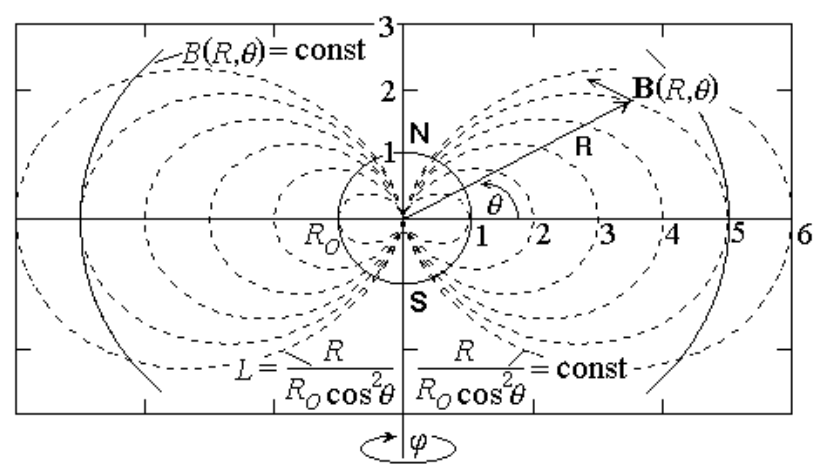

Fig. 1. Dipole magnetic field configuration for the inner Earth's magnetosphere.

cyclotron frequency, in particular, to describe the influence of the drift effects, the finite beta and the finite Larmor radius corrections on the low and ultra-low frequency geomagnetic pulsations in the Earth's magnetosphere (Southwood et al., 1969; Karpman et al., 1977; Chen and Hasegawa, 1991; Klimushkin, 1998; Dettrick et al., 2003). However, to describe the cyclotron effects (for example, by the contribution of the resonant particles to the transverse dielectric permittivity for electromagnetic waves at the fundamental cyclotron frequencies), we should solve the more general Vlasov equation for the perturbed distribution functions in the six-dimensional phase volume, using, for example, an approach developed by Grishanov et al. (2004).

\section{Dipole magnetospheric plasma with the bi- Maxwellian distribution}

At first, let us derive the contribution of energetic particles with anisotropic temperature to the transverse dielectric tensor components for field-aligned cyclotron waves in a dipole magnetospheric plasma, see Fig. 1, where the module of the geomagnetic field, in the spherical coordinates $(R, \theta, \varphi)$, is

$B(R, \theta)=B_{0}\left(\frac{R_{0}}{R}\right)^{3} \sqrt{1+3 \sin ^{2} \theta}$.

Here, $R_{0}$ is the radius of the Earth, $R$ is the geocentric distance, $\theta$ is the geographical latitude, $B_{0}$ is the Earth's equatorial magnetic field, i.e. at the point $R=R_{0}$ and $\theta=0$. The dotted lines in Fig. 1 correspond to the point dipole magnetic field lines $R / R_{0} \cos ^{2} \theta=$ const; and the solid curves $B(R, \theta)=$ const, are corresponding to the lines of the constant geomagnetic field.

To solve the Vlasov equation for collisionless trapped particles with anisotropic temperature we use a standard method of switching to new variables associated with the conservation integrals of the energy: $v_{\|}^{2}+v_{\perp}^{2}=$ const, the magnetic moment: $v_{\perp}^{2} /(2 B)=$ const, and the B-field line equation: $R / \cos ^{2} \theta=$ const . Introducing the variables

$v=\sqrt{v_{\|}^{2}+v_{\perp}^{2}}$,

$\mu=\frac{v_{\perp}^{2} \cos ^{6} \theta}{v^{2} \sqrt{1+3 \sin ^{2} \theta}}$

$L=\frac{R}{R_{0} \cos ^{2} \theta}$

(instead of $v_{\|}, v_{\perp}, R$ ), the perturbed distribution function can be found as

$$
\begin{aligned}
& f\left(t, R, \theta, \varphi, v_{\|}, v_{\perp}, \alpha\right)= \\
& \quad \sum_{s}^{ \pm 1} \sum_{l}^{ \pm \infty} f_{l}^{s}(\theta, L, v, \mu) \exp (-i \omega t+i m \varphi+i l \alpha),
\end{aligned}
$$

where $\alpha$ is the gyrophase angle in velocity space, $v$ is the module of the particle velocity associated with the particle energy, $\mu$ is the nondimensional magnetic moment associated with the pitch angle, and $L$ is the nondimensional $L$ shell parameter, i.e. the shell distance at the equatorial plane, normalized to the Earth's radius. By the indexes $s= \pm 1$ we distinguish the particles with positive and negative values of the parallel velocity relative to $\mathbf{B}$ :

$v_{\|}=s v \sqrt{1-\mu b(\theta)}$.

The linearized Vlasov equation for (interesting us) harmonics $f_{ \pm 1}^{s}$ can be rewritten in the form

$$
\begin{aligned}
& \frac{\sqrt{1-\mu b(\theta)}}{\cos \theta \sqrt{1+3 \sin ^{2} \theta}} \frac{\partial f_{l}^{s}}{\partial \theta}-i s \frac{R_{0} L}{v}\left[\omega+l \frac{\omega_{c o}}{L^{3}} b(\theta)\right] f_{l}^{s}=Q_{l}^{s} \\
& \quad l= \pm 1
\end{aligned}
$$

where

$$
\begin{aligned}
& Q_{l}^{s}=\frac{e R_{0} L}{M v_{T \|}^{2}} \sqrt{\mu} F_{0} \times \\
& {\left[s \frac{E_{l}}{\sqrt{b(\theta)}}\left[b(\theta)-1+\frac{T_{\|}}{T_{\perp}}\right]-\frac{i v \sqrt{1-\mu b(\theta)}\left(1-T_{\|} / T_{\perp}\right)}{\omega R_{0} L \cos \theta \sqrt{1+3 \sin ^{2} \theta}} \frac{\partial}{\partial \theta} \frac{E_{l}}{\sqrt{b(\theta)}}\right],} \\
& F_{0}=\frac{N(L)}{\pi^{1.5} v_{T \|} v_{T \perp}^{2}} \exp \left\{-\frac{v^{2}}{v_{T \|}^{2}}\left[1-\mu\left(1-\frac{T_{\|}}{T_{\perp}}\right)\right]\right\} \\
& v_{T \|}^{2}=\frac{2 T_{\|}}{M}, \quad v_{T \perp}^{2}=\frac{2 T_{\perp}}{M} \\
& b(\theta)=\frac{\sqrt{1+3 \sin ^{2} \theta}}{\cos ^{6} \theta} .
\end{aligned}
$$

Here, $E_{n}$ and $E_{b}$ are, respectively, the normal and binormal perturbed electric field components relative to $\mathbf{B}$; $E_{l}=E_{n}-i l E_{b}$ is the transverse electric field component corresponding to either the right-hand polarized wave, if $l=1$, 
and to left-hand polarized wave, if $l=-1 ; F_{0}$ is the biMaxwellian distribution function of plasma particles with density $N$, parallel and perpendicular temperature $T_{\|}$and $T_{\perp}$, charge $e$ and mass $M$. Note in Eq. (7) that we have neglected the drift corrections, assuming that the wave frequency $\omega$ is much larger than the drift frequency and the Larmor radius of plasma particles is small, which is valid when $m v_{T \perp}^{2} L^{2} /\left(v_{T||} R_{0} \omega_{c o}\right) \ll 1$, where $\omega_{c o}=e B_{0} / M c$ and $m$ is the azimuthal wave number over the $\varphi$ (east-west) direction in an equatorial plane. Moreover, deriving Eq. (7), as well as the dispersion equation for field-aligned cyclotron waves, we assume that the plasma is perfectly conducting, i.e. $E_{\|}=0$, and the left-hand polarized $\left(E_{-1}=E_{n}+i E_{b}\right)$ and right-hand polarized $\left(E_{+1}=E_{n}-i E_{b}\right)$ waves are connected weakly.

Depending on $\mu$ and $\theta$, the domain of the perturbed distribution functions is defined by the inequalities

$\mu_{0} \leq \mu \leq 1 \quad$ and $\quad-\theta_{t}(\mu) \leq \theta \leq \theta_{t}(\mu)$

where

$\mu_{0}=\frac{1}{b(\arccos \sqrt{1 / L})}=\frac{1}{L^{2.5} \sqrt{4 L-3}}$,

and $\pm \theta_{t}(\mu)$ are the local mirror (or turning, or stop, or reflection) points for trapped particles at a given (by $L$ ) magnetic field line, which are defined by the condition $v_{\|}\left( \pm \theta_{t}\right)=0$. Any untrapped particle with $\mu \leq \mu_{0}$ cannot survive more than one-half of the bounce time and will be precipitated into the atmosphere/ionosphere.

Since the trapped particles, with a given (by $\mu$ ) pitch angle, execute the bounce-periodic motion, the solution of Eq. (7) (in the zeroth order of a magnetization parameter) can be found as

$f_{l}^{s}(\theta, L, v, \mu)=$

$\sum_{p=-\infty}^{+\infty} f_{l}^{s}(L, v, \mu) \exp \left[i p \frac{2 \pi}{\tau_{b}} \tau(\theta)+i s l \frac{R_{0} \omega_{c o}}{L^{2} v} C(\theta)\right]$,

where

$\tau(\theta)=\int_{0}^{\theta} \frac{\cos \eta \sqrt{1+3 \sin ^{2} \eta}}{\sqrt{1-\mu b(\eta)}} d \eta$

$C(\theta)=\int_{0}^{\theta} b(\eta) \frac{\cos \eta \sqrt{1+3 \sin ^{2} \eta}}{\sqrt{1-\mu b(\eta)}} d \eta-\bar{b} \tau(\theta)$,

$\bar{b}=\frac{4}{\tau_{b}} \int_{0}^{\theta_{t}} b(\theta) \frac{\cos \theta \sqrt{1+3 \sin ^{2} \theta}}{\sqrt{1-\mu b(\theta)}} d \theta$.

The perturbed distribution functions, in the form of Eq. (14), satisfy automatically the corresponding boundary conditions for the trapped particles, namely, the continuity of the distribution functions at the reflection points $\pm \theta_{t}$ :

$f_{l}^{+1}\left( \pm \theta_{t}\right)=f_{l}^{-1}\left( \pm \theta_{t}\right)$ or the same

$f_{l}^{s}(\tau(\theta))=f_{l}^{s}\left(\tau(\theta)+\tau_{b}\right)$,

where $\tau_{b}=\tau_{b}(\mu)=4 \tau\left(\theta_{t}\right)$ is the normalized bounce period of the trapped particles. In our notations, the bounce frequency of the trapped particles with a given temperature $T_{\|}$and pitch angle $\mu$ is defined as

$\omega_{b}=\frac{2 \pi v_{T \|}}{R_{0} L \tau_{b}}$

After solving Eq. (7), the contribution of the trapped particles to the two-dimensional transverse current density component, $j_{ \pm 1}(\theta, L)$, can be expressed as

$j_{l}(\theta, L)=\frac{\pi e}{2} b^{1.5}(\theta) \sum_{s}^{ \pm 1} \int_{0}^{\infty} v^{3} d v \int_{\mu_{0}}^{1 / b(\theta)} \frac{\sqrt{\mu} f_{l}^{s}(\theta, L, v, \mu)}{\sqrt{1-\mu b(\theta)}} d \mu$ $l= \pm 1$.

Note that the normal and binormal (to B) current density components in our notation are equal, respectively, to

$j_{n}=j_{+1}+j_{-1} \quad$ and $\quad j_{b}=i\left(j_{+1}-j_{-1}\right)$.

To solve the wave (or Maxwell's) equations it is convenient to expand the perturbed values in a Fourier series over the coordinate $\lambda$ along the geomagnetic field line

$\lambda(\theta)=$

$\frac{R_{0} L}{2 \sqrt{3}}\left[\sqrt{3} \sin \theta \sqrt{1+3 \sin ^{2} \theta}+\ln \left(\sqrt{3} \sin \theta+\sqrt{1+3 \sin ^{2} \theta}\right)\right]$

measured from the equator. In particular, for the transverse components of the current density, $j_{l}$, and electric field, $E_{l}$, we have:

$\frac{j_{l}(\theta, L)}{\sqrt{b(\theta)}}=\sum_{n}^{ \pm \infty} j_{l}^{(n)}(L) \exp \left[i \pi n \frac{\lambda(\theta)}{\lambda_{o}}\right]$

$\frac{E_{l}(\theta, L)}{\sqrt{b(\theta)}}=\sum_{n^{\prime}}^{ \pm \infty} E_{l}^{\left(n^{\prime}\right)}(L) \exp \left[i \pi n^{\prime} \frac{\lambda(\theta)}{\lambda_{o}}\right]$,

where $\lambda_{o}=\lambda(\arccos \sqrt{1 / L})$ is the half-length of a given by $L$ magnetic field line. This procedure converts the operator, representing the dielectric tensor, into a matrix whose elements can be calculated independently of the solutions of Maxwell's equations. As a result, there is the following connection for harmonics $j_{l}^{(n)}$ and $E_{l}^{\left(n^{\prime}\right)}$ :

$\frac{4 \pi i}{\omega} j_{l}^{(n)}(L)=\sum_{n^{\prime}}^{ \pm \infty} \varepsilon_{l}^{n, n^{\prime}}(L) \cdot E_{l}^{\left(n^{\prime}\right)}(L)$,

and the contribution of a given kind of plasma particles to the transverse permittivity elements, $\varepsilon_{l}^{n, n^{\prime}}(L)$, after the $s$-summation, is

$\varepsilon_{l}^{n, n^{\prime}}=\sum_{p=-\infty}^{\infty} \varepsilon_{l, p}^{n, n^{\prime}}$,

where 
$\varepsilon_{l, p}^{n, n^{\prime}}=\frac{\omega_{p o}^{2} L^{2} R_{0}^{2} T_{\|}}{8 \omega \pi^{1.5} \lambda_{o} v_{T \|} T_{\perp}} \int_{\mu_{0}}^{1} \mu d \mu \int_{0}^{\infty} u^{4} \frac{D_{p, l}^{n}(u, \mu) G_{p, l}^{n^{\prime}}(u, \mu)}{p u-Z_{l}(\mu)} \exp \left[-u^{2}\left(1-\mu\left(1-\frac{T_{\|}}{T_{\perp}}\right)\right)\right] d u$

is the separate contribution of the $p$-bounce resonant term to $\varepsilon_{l}^{n, n^{\prime}}(L)$, and the other definitions are

$$
\begin{aligned}
& \omega_{p o}^{2}=\frac{4 \pi N e^{2}}{M}, \quad u=\frac{v}{v_{T \|}}, \quad Z_{l}(\mu)=\frac{1}{\omega_{b}}\left(\omega+l \frac{\omega_{c o}}{L^{3}} \bar{b}\right) \\
& G_{p, l}^{n}(u, \mu)=\int_{-\theta_{t}}^{\theta_{t}}\left(b(\theta)-1+\frac{T_{\|}}{T_{\perp}}+\frac{\pi n u v_{T} \|}{\omega \lambda_{o}}\left(1-\frac{T_{\|}}{T_{\perp}}\right) \sqrt{1-\mu b(\theta)}\right) \cos \left[\frac{n \pi}{\lambda_{o}} \lambda(\theta)-p \frac{2 \pi}{\tau_{b}} \tau(\theta)-\frac{l R_{0} \omega_{c o}}{L^{2} u v_{T} \|} C(\theta)\right] \frac{\cos \theta \sqrt{1+3 \sin ^{2} \theta}}{\sqrt{1-\mu b(\theta)}} d \theta \\
& +(-1)^{p} \int_{-\theta_{t}}^{\theta_{t}}\left(b(\theta)-1+\frac{T_{\|}}{T_{\perp}}+\frac{\pi n u v_{T \|}}{\omega \lambda_{o}}\left(1-\frac{T_{\|}}{T_{\perp}}\right) \sqrt{1-\mu b(\theta)}\right) \cos \left[\frac{n \pi}{\lambda_{o}} \lambda(\theta)+p \frac{2 \pi}{\tau_{b}} \tau(\theta)+\frac{l R_{0} \omega_{c o}}{L^{2} u v_{T \|}} C(\theta)\right] \frac{\cos \theta \sqrt{1+3 \sin ^{2} \theta}}{\sqrt{1-\mu b(\theta)}} d \theta,(30) \\
& D_{p, l}^{n}(u, \mu)=\int_{-\theta_{t}}^{\theta_{t}} \cos \left[\frac{n \pi}{\lambda_{o}} \lambda(\theta)-p \frac{2 \pi}{\tau_{b}} \tau(\theta)-\frac{l R_{0} \omega_{c o}}{L^{2} u v_{T \|}} C(\theta)\right] b(\theta) \frac{\cos \theta \sqrt{1+3 \sin ^{2} \theta}}{\sqrt{1-\mu b(\theta)}} d \theta \\
& +(-1)^{p} \int_{-\theta_{t}}^{\theta_{t}} \cos \left[\frac{n \pi}{\lambda_{o}} \lambda(\theta)+p \frac{2 \pi}{\tau_{b}} \tau(\theta)+\frac{l R_{0} \omega_{c o}}{L^{2} u v_{T \|}} C(\theta)\right] b(\theta) \frac{\cos \theta \sqrt{1+3 \sin ^{2} \theta}}{\sqrt{1-\mu b(\theta)}} d \theta
\end{aligned}
$$

Thus, the transverse permittivity elements for electromagnetic waves (at the fundamental cyclotron frequency) in an axisymmetric dipole magnetospheric plasma are expressed by the $p$-summation of the bounce-resonant terms, including the double integration in velocity space, the resonant denominators $p u-Z_{l}(\mu)$, and the phase coefficients $G_{p, l}^{n}(u, \mu)$ and $D_{p, l}^{n}(u, \mu)$. As follows from Eq. (26), due to a 2-D geomagnetic field nonuniformity, the whole spectrum of the electric field (by $\Sigma_{n^{\prime}}^{ \pm \infty}$ ) is present in the given (by $n$ ) current density harmonic. It should be noted that the bounce-resonance conditions, $p u-Z_{l}(\mu)=0$ for trapped particles in magnetospheric plasmas, are different from the corresponding expressions in the straight magnetic field case. Of course, as in the straight magnetic field, $l=1$ corresponds to the effective resonant interaction of electrons with the extraordinary (or right-hand polarized) waves at the fundamental electron-cyclotron frequency, and $l=-1$ corresponds to the resonant interaction of ions with an ordinary (or left-hand polarized) wave at the fundamental ion-cyclotron frequency. Note that it is impossible to carry out the analytical Landau integration over the particle energy $u=v / v_{T \| \mid}$ (by introducing the plasma dispersion functions) because the phase coefficients $G_{p, l}^{n^{\prime}}(u, \mu)$ and $D_{p, l}^{n}(u, \mu)$ depend on $u$. As for the particles with isotropic temperature, i.e. if $T_{\|}=T_{\perp}$, the phase coefficients $G_{p, l}^{n}(u, \mu)$ can be reduced to

$G_{p, l}^{n}(u, \mu)=D_{p, l}^{n}(u, \mu)$.

As was noted above, Eqs. (27-31) describe the contribution of any kind of the trapped particles to the transverse permittivity elements. The corresponding expressions for plasma electrons and ions can be obtained from Eqs. (27-31) by replacing the temperatures $T_{\|}$and $T_{\perp}$, density $N$, mass $M$, charge $e$ by the electron $T_{\| e}, T_{\perp e}, N_{e}, M_{e}, e_{e}$ and ion $T_{\| i}$, $T_{\perp i}, N_{i}, M_{i}, e_{i}$ parameters, respectively.

Since the cyclotron wave instabilities are an important contributor to the geomagnetic storms during the solar activity, it is possible to develop a 2-D numerical code to describe these processes in the Earth's magnetosphere with new dielectric tensor components, accounting for the bounceresonant effects. To have some analogy with linear theory of cyclotron wave instabilities in the straight magnetic field (e.g. Kennel and Petschek, 1966), let us assume that the n-th harmonic of the electric field gives the main contribution to the n-th harmonic of the current density (one-mode approximation). In this case, for the field-aligned electromagnetic cyclotron waves (when $m=0, \partial / \partial L=0, E_{\|}=0, H_{\|}=0$ ), from the Maxwell's equations

$$
E_{l}+\frac{8 \pi i}{\omega} j_{l}=
$$




$$
\frac{-c^{2} \sqrt{b(\theta)}}{\omega^{2} R_{0}^{2} L^{2} \cos \theta \sqrt{1+3 \sin ^{2} \theta}} \frac{\partial}{\partial \theta} \frac{1}{\cos \theta \sqrt{1+3 \sin ^{2} \theta}} \frac{\partial}{\partial \theta} \frac{E_{l}}{\sqrt{b(\theta)}}
$$

excluding the $E_{l}^{(n)}$-harmonics by Eqs. (24-26), we obtain the following dispersion equation:

$$
\left(\frac{n \pi c}{\lambda_{o} \omega}\right)^{2}=1+2 \sum_{\sigma} \varepsilon_{l,(\sigma)}^{n, n}(L)
$$

where $\sigma$ denotes the particle species (electron, proton, heavy ions). This equation is suitable to analyze the instability of the right-hand polarized waves, if $l=1$, and the left-hand polarized waves, if $l=-1$. Note that, in our notation, the parallel wave vector is defined as $k_{\|}=n \pi / \lambda_{o}$, so that $n \pi c /\left(\lambda_{o} \omega\right)$ is the nondimensional parallel refractive index. Further, Eq. (34) should be resolved numerically for the real and imaginary parts of the wave frequency, $\omega=\operatorname{Re} \omega+i \operatorname{Im} \omega$, to define the conditions of the wave instabilities in the dipole magnetospheric plasmas with anisotropic temperature. As usual, the growth (damping) rate of the electromagnetic cyclotron waves, $\operatorname{Im} \omega$, is defined by the contribution of the resonant particles to the imaginary part of the transverse permittivity elements, $\operatorname{Im} \varepsilon_{l,(\sigma)}^{n, n}$, that can be readily derived from Eqs. (27) and (28), using the well-known residue (or Landau rule) method.

\section{Dipole magnetospheric plasma with the bi-Lorentzian distribution}

Of course, an approach developed in the previous section for magnetospheric plasmas with bi-Maxwellian distribution functions in velocity space can be applied, as well for plasmas with the more general distributions including, for example, the bi-Lorentzian distribution functions. According to Summers and Thorne (1991), Chan et al. (1994), Xue et al. (1996), the generalised steady-state bi-Lorentzian (or kappa) distribution functions can be expressed as

$$
F_{0}=\frac{N(L)}{\pi^{1.5} \vartheta_{\|} \vartheta_{\perp}^{2} \kappa^{1.5}} \frac{\Gamma(1+\kappa)}{\Gamma(\kappa-0.5)}\left\{1+\frac{v^{2}}{\kappa \vartheta_{\|}^{2}}\left[1-\mu\left(1-\frac{T_{\|}}{T_{\perp}}\right)\right]\right\}^{-(1+\kappa)}
$$

with the associated effective thermal speeds corresponding to $T_{\|}$and $T_{\perp}$,

$$
\vartheta_{\|}^{2}=\frac{2 \kappa-3}{\kappa} \frac{T_{\|}}{M}, \quad \vartheta_{\perp}^{2}=\frac{2 \kappa-3}{\kappa} \frac{T_{\perp}}{M},
$$

where the parameter $\kappa$ is the spectral index (here it takes positive values $\kappa \geq 2) ; \Gamma(x)=\int_{0}^{\infty} t^{x-1} \exp (-t) d t$ is the gamma function. It should be noted that the parameter $\kappa$ is a measure of the proportion of the high energy particles present in the distribution; typically for the space plasmas, it is found to be in the range $2 \leq \kappa \leq 6$. Moreover, the generalised biLorentzian distribution contains the standard bi-Maxwellian distribution, Eq. (9), as a special case letting $\kappa \rightarrow \infty$.

To estimate the main contribution of the trapped particles with the bi-Lorentzian distribution functions to the transverse permittivity elements we should solve again Eq. (7), where the right-hand side is

$$
\hat{Q}_{l}^{s}=\frac{e R_{0} L \sqrt{\mu}(1+\kappa) F_{0}}{M \kappa \vartheta_{\|}^{2}\left[1+\frac{v^{2}}{\kappa \vartheta_{\|}^{2}}\left[1-\mu\left(1-\frac{T_{||}}{T_{\perp}}\right)\right]\right]}\left[\frac{s E_{l}}{\sqrt{b(\theta)}}\left[b(\theta)-1+\frac{T_{||}}{T_{\perp}}\right]-\frac{i v \sqrt{1-\mu b(\theta)}\left(1-T_{||} / T_{\perp}\right)}{\omega R_{0} L \cos \theta \sqrt{1+3 \sin ^{2} \theta}} \frac{\partial}{\partial \theta} \frac{E_{l}}{\sqrt{b(\theta)}}\right] .
$$

As a result, one can derive the new expression $\varepsilon_{l}^{n, n^{\prime}}(L)$ for field-aligned waves in magnetospheric plasmas with the biLorentzian distribution functions in velocity space, instead of Eqs. (27-31):

$$
\varepsilon_{l}^{n, n^{\prime}}=\frac{\omega_{p o}^{2} L R_{0} T_{\|}(\kappa+1) \Gamma(\kappa+1)}{8 \omega \pi^{1.5} \lambda_{o} T_{\perp} \sqrt{\kappa} \vartheta_{\|} \Gamma(\kappa-0.5)} \sum_{p=-\infty}^{\infty} \int_{\mu_{0}}^{1} \mu d \mu \int_{-\infty}^{\infty} \frac{u^{4} \hat{D}_{p, l}^{n}(u, \mu) \hat{G}_{p, l}^{n^{\prime}}(u, \mu) d u}{\left[p u-\hat{Z}_{l}(\mu)\right]\left[1+u^{2}\left(1-\mu\left(1-\frac{T_{\|}}{T_{\perp}}\right)\right)\right]^{\kappa+2}},
$$

where

$$
\begin{aligned}
& \hat{G}_{p, l}^{n}(u, \mu)=\int_{-\theta_{t}}^{\theta_{t}}\left(b(\theta)-1+\frac{T_{\|}}{T_{\perp}}+\frac{\pi n u \sqrt{\kappa} \vartheta_{\|}}{\omega \lambda_{o}}\left(1-\frac{T_{\|}}{T_{\perp}}\right) \sqrt{1-\mu b(\theta)}\right) \cos \left[\frac{n \pi}{\lambda_{o}} \lambda(\theta)-p \frac{2 \pi}{\tau_{b}} \tau(\theta)-\frac{l R_{0} \omega_{c o}}{L^{2} u \sqrt{\kappa} \vartheta_{\|}} C(\theta)\right] \frac{\cos \theta \sqrt{1+3 \sin ^{2} \theta}}{\sqrt{1-\mu b(\theta)}} d \theta \\
& +(-1)^{p} \int_{-\theta_{t}}^{\theta_{t}}\left(b(\theta)-1+\frac{T_{\|}}{T_{\perp}}+\frac{\pi n u \sqrt{\kappa} \vartheta_{\|}}{\omega \lambda_{o}}\left(1-\frac{T_{\|}}{T_{\perp}}\right) \sqrt{1-\mu b(\theta)}\right) \cos \left[\frac{n \pi}{\lambda_{o}} \lambda(\theta)+p \frac{2 \pi}{\tau_{b}} \tau(\theta)+\frac{l R_{0} \omega_{c o}}{L^{2} u \sqrt{\kappa} \vartheta_{\|}} C(\theta)\right] \frac{\cos \theta \sqrt{1+3 \sin ^{2} \theta}}{\sqrt{1-\mu b(\theta)}} d \theta, \quad(39) \\
& \hat{D}_{p, l}^{n}(u, \mu)=\int_{-\theta_{t}}^{\theta_{t}} \cos \left[\frac{n \pi}{\lambda_{o}} \lambda(\theta)-p \frac{2 \pi}{\tau_{b}} \tau(\theta)-\frac{l R_{0} \omega_{c o}}{L^{2} u \sqrt{\kappa} \vartheta_{\|}} C(\theta)\right] b(\theta) \frac{\cos \theta \sqrt{1+3 \sin ^{2} \theta}}{\sqrt{1-\mu b(\theta)}} d \theta
\end{aligned}
$$


$+(-1)^{p} \int_{-\theta_{t}}^{\theta_{t}} \cos \left[\frac{n \pi}{\lambda_{o}} \lambda(\theta)+p \frac{2 \pi}{\tau_{b}} \tau(\theta)+\frac{l R_{0} \omega_{c o}}{L^{2} u \sqrt{\kappa} \vartheta_{\|}} C(\theta)\right] b(\theta) \frac{\cos \theta \sqrt{1+3 \sin ^{2} \theta}}{\sqrt{1-\mu b(\theta)}} d \theta$,

$u=\frac{v}{\sqrt{\kappa} \vartheta_{\|}}, \quad \hat{Z}_{l}(\mu)=\frac{R_{0} L \tau_{b}}{2 \pi \sqrt{\kappa} \vartheta_{\|}}\left(\omega+l \frac{\omega_{c o}}{L^{3}} \bar{b}\right)$.

It should be noted that, in the variables $(v, \mu)$, there are identities for the phase coefficients: $G_{p, l}^{n}(v, \mu)=\hat{G}_{p, l}^{n}(v, \mu)$ and $D_{p, l}^{n}(v, \mu)=\hat{D}_{p, l}^{n}(v, \mu)$. Accordingly, the single waveparticle resonance conditions are also independent of the steady-state distribution functions of the trapped particles and can be written as

$\omega+l \frac{\omega_{c o}}{L^{3}} \bar{b}(\mu)=p \frac{2 \pi_{v}}{R_{0} L \tau_{b}(\mu)}$,

involving the wave frequency $\omega$, the bounce-averaged cyclotron frequency $\omega_{c o} \bar{b}(\mu) / L^{3}$ and the bounce frequency of the trapped particles $2 \pi v /\left[R_{0} L \tau_{b}(\mu)\right]$, with the given energy $v$ and pitch angle $\mu$ at the given (by $L$ ) magnetic field line, where $l=0, \pm 1, \ldots$ and $p=0, \pm 1, \ldots$ are the numbers of the cyclotron and bounce resonances, respectively.

In the case of the bi-Lorentzian distribution functions, the dispersion equation for the cyclotron waves will be the same as Eq. (34), where the new transverse dielectric tensor components, Eq. (38), should be included. For specified values of the ambient magnetic field line $L$, and the parameters $\kappa$ $T_{\| \sigma}, T_{\perp \sigma}, N_{\sigma}, M_{\sigma}, e_{\sigma}$ that describe each particle species, an iterative scheme can be used to solve Eq. (34) for a com- plex wave frequency, for prescribed real values of the wave number $k_{\|}=n \pi / \lambda_{o}$.

\section{Magnetospheric plasma with circular magnetic field lines (bi-Maxwellian distribution)}

By analogy, we can derive the contribution of the resonance particles to the transverse dielectric permittivity for waves in magnetospheric plasmas with circular magnetic field lines, where the module of the magnetic field is

$B=B_{0}\left(\frac{R_{0}}{R}\right)^{3} \frac{1}{\cos \theta}=\frac{B_{0}}{L^{3} \cos ^{4} \theta}$.

Here the new $L$-shell variable is introduced instead of $R$ as $L=R /\left(R_{0} \cos \theta\right)$, taking into account that the equation of the B-field lines, in this case, has a form $R / \cos \theta=$ const, and the nondimensional parameter $b(\theta)$ is

$b(\theta)=\frac{B(L, \theta)}{B(L, 0)}=\frac{1}{\cos ^{4} \theta}$.

In this case, the linearized Vlasov equation for harmonics $f_{l}$ can be reduced to

$\sqrt{1-\mu b(\theta)} \frac{\partial f_{l}^{s}}{\partial \theta}-i s \frac{R_{0} L}{v}\left[\omega+l \frac{\omega_{c o}}{L^{3}} b(\theta)\right] f_{l}^{s}=Q_{l}^{s}, \quad l= \pm 1$,

where

$Q_{l}^{s}=\frac{e R_{0} L}{M v_{T \|}^{2}} \sqrt{\mu} F_{0}\left[s \frac{E_{l}}{\sqrt{b(\theta)}}\left(b(\theta)-1+\frac{T_{\|}}{T_{\perp}}\right)-i \frac{v \sqrt{1-\mu b(\theta)}}{\omega R_{0} L c}\left(1-\frac{T_{\|}}{T_{\perp}}\right) \frac{\partial}{\partial \theta}\left(\frac{E_{l}}{\sqrt{b(\theta)}}\right)\right]$.

In contrast to a dipole geomagnetic field, the length element along the magnetic field line in magnetospheric plasmas with circular magnetic field lines is proportional to the latitude angle (as a linear function), i.e. $\lambda_{c}(\theta)=R_{0} L \theta$, and the new time-like variable $\tau$ can be introduced by the elliptic integrals

$\tau(\theta, v)=\sqrt{\frac{2(1-v)^{2}}{1-2 v}} \Pi\left(\arcsin \left(\sqrt{\frac{1-2 v}{2(1-v)-\sin ^{2} \theta}} \sin \theta\right), \frac{-v}{1-2 v}, v\right)-\sqrt{\frac{1-2 v}{2}} F\left(\arcsin \left(\sqrt{\frac{1-2 v}{2(1-v)-\sin ^{2} \theta}} \sin \theta\right), v\right)$,

where

$\Pi(\alpha, \delta, v)=\int_{0}^{\alpha} \frac{d \eta}{\left(1-\delta \sin ^{2} \eta\right) \sqrt{1-v \sin ^{2} \eta}}$ and

$$
F(\alpha, v)=\int_{0}^{\alpha} \frac{d \eta}{\sqrt{1-v \sin ^{2} \eta}}
$$

are the incomplete elliptic integrals of the third and first kind, respectively. Accordingly, the bounce periods of the trapped 
particles (normalized to $R_{0} L / v_{T||}$ ) can be expressed by the complete elliptic integral of the third kind as

$T_{b}(\nu)=2 \sqrt{2(1-2 v)} \Pi(\pi / 2,2 \nu, v)$,

where the $v$ variable is introduced instead of $\mu$ as $\mu=(1-2 v)^{2}$, varying in the interval

$0 \leq v=0.5(1-\sqrt{\mu}) \leq \frac{L^{2}-1}{2 L^{2}}$.

Here we have taken into account that, in the case of the circular magnetic field lines, the reflection points for the trapped particles are defined by the simple relation

$\theta_{t}(v)=\arcsin \sqrt{2 v}$,

and the maximal $\theta_{t}(v)$ for trapped particles with the given

$v_{o}=\frac{L^{2}-1}{2 L^{2}}$,

at the $L$-shell is

$\theta_{o}=\theta_{t}\left(v_{o}\right)=\arccos \left(\frac{1}{L}\right)$.
As a result, using the Fourier expansions

$$
\begin{aligned}
& \frac{j_{l}(\theta, L)}{\sqrt{b(\theta)}}=\sum_{n}^{ \pm \infty} j_{l}^{(n)}(L) \exp \left[i \pi n \frac{\theta}{\theta_{o}}\right], \\
& \frac{E_{l}(\theta, L)}{\sqrt{b(\theta)}}=\sum_{n^{\prime}}^{ \pm \infty} E_{l}^{\left(n^{\prime}\right)}(L) \exp \left[i \pi n^{\prime} \frac{\theta}{\theta_{o}}\right]
\end{aligned}
$$

the contribution of the resonant particles to $\varepsilon_{l}^{n, n^{\prime}}(L)$ :

$$
\frac{4 \pi i}{\omega} j_{l}^{(n)}(L)=\sum_{n^{\prime}}^{ \pm \infty} \varepsilon_{l}^{n, n^{\prime}}(L) \cdot E_{l}^{\left(n^{\prime}\right)}(L)
$$

can be expressed as

$$
\varepsilon_{l}^{n, n^{\prime}}=\sum_{p=-\infty}^{\infty} \varepsilon_{l, p}^{n, n^{\prime}}
$$

where

$\varepsilon_{l, p}^{n, n^{\prime}}=\frac{\omega_{p o}^{2} L R_{0} T_{\|}}{2 \omega \pi^{1.5} \theta_{o} v_{T \|}^{T_{\perp}}} \int_{0}^{v_{o}}(1-2 v)^{3} d v \int_{0}^{\infty} \frac{u^{4} A_{p, l}^{n} Y_{p, l}^{n^{\prime}}}{p u-Z_{l}(v)} \exp \left[-u^{2}\left(1-(1-2 v)^{2}\left(1-\frac{T_{\|}}{T_{\perp}}\right)\right)\right] d u$

and the phase coefficients are

$$
\begin{aligned}
& Y_{p, l}^{n}(u, v)=\int_{-\theta_{t}}^{\theta_{t}}\left(b(\theta)-1+\frac{T_{\|}}{T_{\perp}}+\frac{\pi n u v_{T \|}}{\omega R_{0} L \theta_{o}}\left(1-\frac{T_{\|}}{T_{\perp}}\right) \sqrt{1-(1-2 v)^{2} b(\theta)}\right) \cos \left[\frac{n \pi}{\theta_{o}} \theta-p \frac{2 \pi}{\tau_{b}} \tau(\theta)-\frac{l R_{0} \omega_{c o}}{L^{2} u v_{T \|}} C(\theta)\right] \frac{d \theta}{\sqrt{1-(1-2 v)^{2} b(\theta)}}+ \\
& (-1)^{p} \int_{-\theta_{t}}^{\theta_{t}}\left(b(\theta)-1+\frac{T_{\|}}{T_{\perp}}+\frac{\pi n u v_{T \|}}{\omega R_{0} L \theta_{o}}\left(1-\frac{T_{\|}}{T_{\perp}}\right) \sqrt{1-(1-2 v)^{2} b(\theta)}\right) \cos \left[\frac{n \pi}{\theta_{o}} \theta+p \frac{2 \pi}{\tau_{b}} \tau(\theta)+\frac{l R_{0} \omega_{c o}}{L^{2} u v_{T} \|} C(\theta)\right] \frac{d \theta}{\sqrt{1-(1-2 \nu)^{2} b(\theta)}}, \\
& A_{p, l}^{n}(u, v)=\int_{-\theta_{t}}^{\theta_{t}} \cos \left[\frac{n \pi}{\theta_{o}} \theta-p \frac{2 \pi}{\tau_{b}} \tau(\theta)-\frac{l R_{0} \omega_{c o}}{L^{2} u v_{T \|}} C(\theta)\right] \frac{b(\theta) d \theta}{\sqrt{1-(1-2 \nu)^{2} b(\theta)}}+ \\
& (-1)^{p} \int_{-\theta_{t}}^{\theta_{t}} \cos \left[\frac{n \pi}{\theta_{o}} \theta+p \frac{2 \pi}{\tau_{b}} \tau(\theta)+\frac{l R_{0} \omega_{c o}}{L^{2} u v_{T \|}} C(\theta)\right] \frac{b(\theta) d \theta}{\sqrt{1-(1-2 \nu)^{2} b(\theta)}} \\
& C(\theta)=\frac{\sqrt{2}(1-v)}{(1-2 v)^{1.5}} \Pi\left(\arcsin \left(\sqrt{\frac{1-2 v}{2(1-v)-\sin ^{2} \theta}} \sin \theta\right), v, v\right)- \\
& \frac{1}{\sqrt{2}(1-2 v)^{1.5}} F\left(\arcsin \left(\sqrt{\frac{1-2 v}{2(1-v)-\sin ^{2} \theta}} \sin \theta\right), v\right) \text {. }
\end{aligned}
$$


In this case, the Maxwell's equations for the transverse electric field and current density components can be reduced to

$E_{l}+\frac{8 \pi i}{\omega} j_{l}=\frac{-c^{2} \sqrt{b(\theta)}}{\omega^{2} R_{0}^{2} L^{2}} \frac{\partial^{2}}{\partial \theta^{2}} \frac{E_{l}}{\sqrt{b(\theta)}}$,

and excluding $E_{l}^{(n)}$-harmonics by Eqs. (55-57) we have derived the dispersion equation

$$
\left(\frac{n \pi c}{\omega R_{0} L \theta_{o}}\right)^{2}=1+2 \sum_{\sigma} \varepsilon_{l,(\sigma)}^{n, n}(L) .
$$

As was mentioned above, $\varepsilon_{l,(\sigma)}^{n, n}$, in the magnetospheric plasmas with circular magnetic field lines, is simpler than that for a dipolar magnetosphere, since there is the exact expression for the reflection points, $\theta_{t}(v)=\arcsin \sqrt{2 v}$, and the phase coefficients can be calculated using the elliptic inegrals and Jacobi elliptic functions, which are convenient for computations by the last versions of such mathematical programmes as Maple, Mathematica and Mathcad, operating the complete and incomplete elliptic integrals and elliptic functions in the standard forms, such as the usual elementary functions. In particular, the new variable $\alpha$ can be introduced instead of $\theta$ by the transformation

$\theta(\alpha)=\arcsin \left(\sqrt{\frac{2 v(1-v)}{1-2 v+v \operatorname{sn}^{2}(\alpha, \sqrt{v})}} \operatorname{sn}(\alpha, \sqrt{v})\right)$

or

$$
\alpha(\theta)=\int_{0}^{\arcsin \left(\sqrt{\frac{1-2 v}{2(1-v)-\sin ^{2} \theta}} \sin \theta\right)} \frac{d \eta}{\sqrt{1-v \sin ^{2} \eta}},
$$

where $\operatorname{sn}(\alpha, \sqrt{v})$ is the Jacobi elliptic function (e.g. Abramovitz and Stegun, 1972).

\section{Magnetized plasma in the straight magnetic field (bi- Maxwellian distribution)}

In the straight magnetic field case, the transverse dielectric tensor component for field-aligned cyclotron waves is

$\varepsilon_{l, \sigma}=\frac{\omega_{p o, \sigma}^{2}}{2 \omega^{2}}\left[\frac{T_{\perp, \sigma}}{T_{\|, \sigma}}-1+\left(\frac{T_{\perp, \sigma}}{T_{\|, \sigma}}-1\right) \frac{\omega-l \Omega_{c o, \sigma}}{k_{\| \mid} v_{T \|, \sigma}} Z\left(\frac{\omega-l \Omega_{c o, \sigma}}{k_{\|} v_{T \|, \sigma}}\right)+\frac{\omega}{k_{\|} v_{T \|, \sigma}} Z\left(\frac{\omega-l \Omega_{c o, \sigma}}{k_{\|} v_{T \|, \sigma}}\right)\right]$

where the plasma dispersion function $\mathrm{Z}(\zeta)$, Fried and Conte (1961), is associated with the bi-Maxwellian distribution, namely,

$Z(\zeta)=\frac{1}{\sqrt{\pi}} \int_{-\infty}^{\infty} \frac{\exp \left(-t^{2}\right)}{t-\zeta} d t \quad \operatorname{Im} \zeta>0$

with the complex $\zeta$ and with the suitable extension for $\operatorname{Im} \zeta \leq 0$ by analytic continuation.

As a result, the dispersion equation for field-aligned electromagnetic cyclotron waves (see e.g. Abramovitz and Stegun, 1972; Bespalov and Trakhtengerts, 1986; Bittencourt, 1986; Chan et al., 1994; Chen and Hasegawa, 1991; Cornwall, 1965; Cuperman, 1981; Dasso et al., 2002; Dettrick et al., 2003) in magnetized plasmas, confined in the straight magnetic field, has the well-known form (see, e.g. Kennel and Petschek, 1966; Xue et al., 1996) similar to Eqs. (34) and (64)

$$
\left(\frac{k_{\| \mid} c}{\omega}\right)^{2}=1+2 \sum_{\sigma} \varepsilon_{l, \sigma}(L),
$$

where the parallel wave vector component $k_{\|}$is connected with the eigenmode numbers $n$ as $k_{\|}=n \pi / \lambda_{o}$ for the standing waves in the dipolar magnetospheric plasmas, and $k_{\|}=n \pi /\left[R_{0} L \arccos (1 / L)\right]$ for plasmaspheres with circular magnetic field lines.
As is well-known, the squared refractive index of the ioncyclotron waves $(l=-1)$ in the hydrogen plasma, including the electrons and protons, is defined by the expression

$\left(\frac{k_{\| \mid} c}{\operatorname{Re} \omega}\right)^{2} \approx \frac{\Omega_{p p}^{2}}{\Omega_{c 0}\left(\Omega_{c 0}-\operatorname{Re} \omega\right)}$,

where $\Omega_{p p}^{2}=4 \pi N_{p} e^{2} / M_{p}$ is the squared Langmuir frequency of the protons calculated by the sum-density of the cold $\left(N_{c}\right)$ and resonant hot $\left(N_{h}\right)$ protons, i.e. $N_{p}=N_{c}+N_{h}$, and $\Omega_{c 0}=e B(L, 0) / M_{p} c=\omega_{c o, p} / L^{3}$ is the equatorial gyrofrequency of $L$-shell protons, so that the eigenmode numbers $n$ (since $k_{\|}=n \pi / \lambda_{o}$ ) can be estimated as

$n \approx \frac{\lambda_{o} \Omega_{p p} \operatorname{Re} \omega}{\pi c \sqrt{\Omega_{c 0}\left(\Omega_{c 0}-\operatorname{Re} \omega\right)}}$

for dipolar geomagnetic field, and by analogy

$n \approx \frac{R_{0} L \arccos (1 / L) \Omega_{p p} \operatorname{Re} \omega}{\pi c \sqrt{\Omega_{c 0}\left(\Omega_{c 0}-\operatorname{Re} \omega\right)}}$

for the case of circular magnetic field lines. As follows from Eq. (70), the propagation of the electromagnetic ioncyclotron (EMIC) waves is possible in the frequency range $R e \omega<\Omega_{c 0}$. It should be noted that Eqs. (70), (71) and (72) are based upon the cold plasma approximation and may not be strictly valid for hot magnetospheric plasmas. The increment (decrement) $\gamma$ of EMIC waves in the hydrogen plasma, including the energetic protons with anisotropic temperature, 
under the condition $\gamma=\operatorname{Im} \omega \ll \operatorname{Re} \omega$, is defined by the expres- $\quad$ where sion

$\frac{\gamma}{\Omega_{c 0}} \approx-2 \frac{\operatorname{Re} \omega\left(\Omega_{c 0}-\operatorname{Re} \omega\right)^{2}}{\Omega_{p p}^{2}\left(2 \Omega_{c 0}-\operatorname{Re} \omega\right)} \operatorname{Im} \varepsilon_{-1, h}$

$\operatorname{Im} \varepsilon_{-1, h}=\frac{\Omega_{p h}^{2} \Omega_{c 0} \sqrt{\pi}}{2(\operatorname{Re} \omega)^{2} k_{\|} v_{T \| h}}\left[\frac{\operatorname{Re} \omega}{\Omega_{c 0}}-\left(1-\frac{\operatorname{Re} \omega}{\Omega_{c 0}}\right)\left(\frac{T_{\perp h}}{T_{\| h}}-1\right)\right] \exp \left[-\left(\frac{\operatorname{Re} \omega-\Omega_{c 0}}{k_{\|} v_{T \| h}}\right)^{2}\right]$

is the contribution of the hot protons to the transverse dielectric permittivity for the left-hand polarized cyclotron waves $(l=-1)$. By the subscribed index $h$ we denote the corresponding plasma parameters for energetic/hot protons with density $N_{h}$, perpendicular temperature $T_{\perp h}$, thermal velocity of the resonant protons $v_{T \| h}=\sqrt{2 T_{\| h} / M_{p}}$, calculated by the parallel temperature $T_{\| h}$. As follows from Eqs. (73) and (74), for the temporal growth (damping) rate of EMIC waves, the instability (when $\gamma>0$ ) is possible under the conditions $\operatorname{Im} \varepsilon_{-1, h}<0$. As is well-known, this is possible if the resonant protons have the temperature anisotropy and $T_{\perp h}>T_{|| h}$ (for the left-hand polarized waves).

\section{Numerical results}

Now let us compare the growth rates of the PCI instability in the plasmas, confined in the straight magnetic field, $\gamma_{s}$, and in the 2-D axisymmetric magnetosphere, with circular magnetic field lines, $\gamma_{c}$. We parameterize the position along a field line using the geographical latitude $\theta$. In the following magnetic field model, the ionosphere then occurs at $\pm \theta_{0}= \pm \arccos (1 / L)$, where $L$ is the $L$-shell parameter (i.e. the radius at the equator, normalized to the Earth's radius), and the parallel wave vector $\mathrm{k}_{\|}$and eigenmode number $n$ are connected by Eq. (72). In our simulations, $L=6.6$, which roughly corresponds to geosynchronous orbit. For simplicity, a hydrogen plasma is considered, including the cold electrons with $\mathrm{N}_{e}=11 \mathrm{~cm}^{-3}$, the cold protons with $N_{c}=10 \mathrm{~cm}^{-3}$, and the energetic protons with $N_{h}=1 \mathrm{~cm}^{-3}$. The parallel and transverse temperatures of the energetic protons are given equal to $T_{\| h}=10 \mathrm{keV}$ and $T_{\perp h}=30 \mathrm{keV}$, respectively, whereas the temperature of the cold particles is small and isotropic. In this case, the eigenmode numbers $n$ of the field-aligned EMIC waves can be defined by the well-known expression for the wave refractive index (see, e.g. Eq. 70); the corresponding dependence $n(\omega)$ is plotted in Fig. 2 using Eq. (72).

The growth rate $\gamma_{s}$ for EMIC waves in the straight magnetic field plasma is estimated, as usually, by Eqs. (73) and (74). As for $\gamma_{c}$, for EMIC waves in the magnetospheric-like plasma with circular magnetic field lines, we use the expression similar to Eq. (73),

$$
\frac{\gamma_{c}}{\Omega_{c 0}} \approx-2 \frac{\operatorname{Re} \omega\left(\Omega_{c 0}-\operatorname{Re} \omega\right)^{2}}{\Omega_{p p}^{2}\left(2 \Omega_{c 0}-\operatorname{Re} \omega\right)} \operatorname{Im} \varepsilon_{-1, h}^{n, n},
$$

where the imaginary part of the transverse dielectric permittivity is estimated by Eqs. (58) and (59) as

$$
\operatorname{Im} \varepsilon_{-1, h}^{n, n}=\sum_{p=1}^{\infty} \operatorname{Im} \varepsilon_{-1, p, h}^{n, n},
$$

where

$$
\begin{aligned}
& \operatorname{Im} \varepsilon_{-1, p, h}^{n, n}=\frac{\Omega_{p h}^{2} L R_{0} T_{\|}}{2 \omega \sqrt{\pi} \theta_{o} v_{T \|} T_{\perp} p^{5}} \int_{0}^{v_{o}}(1-2 v)^{3} A_{p,-1}^{n}\left(\frac{Z_{-1, h}}{p}, v\right) Y_{p,-1}^{n}\left(\frac{Z_{-1, h}}{p}, v\right) \times \\
& Z_{-1, h}^{4} \exp \left[-\frac{Z_{-1, h}^{2}}{p^{2}}\left(1-(1-2 v)^{2}\left(1-\frac{T_{\|}}{T_{\perp}}\right)\right)\right] d v
\end{aligned}
$$

is the separate contribution of the bounce resonant terms to $\operatorname{Im} \varepsilon_{-1, h}^{n, n}$. In the other words, we assume that the growth rates $\gamma_{s}$ and $\gamma_{c}$ (under the same wave frequency, eigenmode number and bulk parameters) are different by the different contributions of the energetic protons to the imaginary part of the transverse dielectric permittivity for EMIC waves in the considered plasma models.
The temporal growth rates of the PCI instability versus $\omega$ are present in Fig. 3a for EMIC waves in the straight magnetic field plasma by Eqs. $(73,74)$, and in Fig. $3 b$ for EMIC waves in the 2-D plasmasphere with circular magnetic field lines by Eqs. (75-77). The computations of $\gamma_{c}$ are carried out in the interval $2 \mathrm{~Hz} \leq \omega \leq 7 \mathrm{~Hz}$, whereas the minimal gyrofrequency of the protons at $L=6.6$ is closed to $\Omega_{c 0} \approx 11 \mathrm{~Hz}$. 


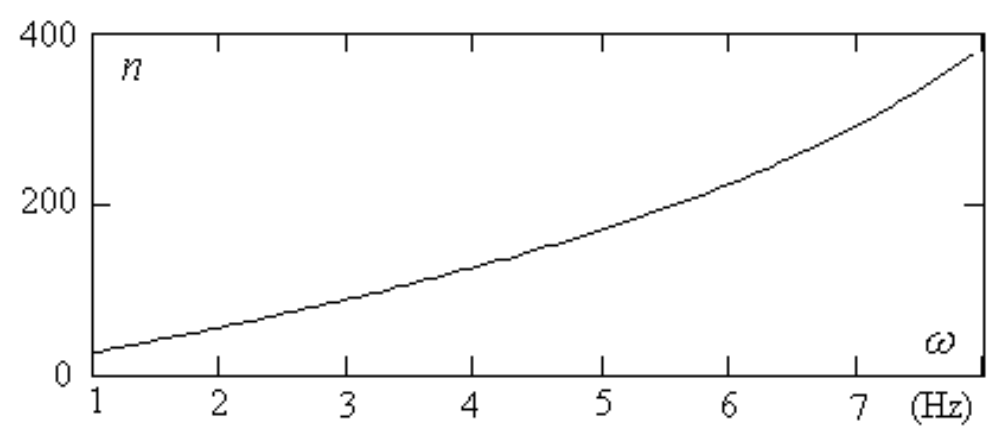

Fig. 2. Dependence of the mode number $n$ on the wave frequency $\omega$ or left-hand polarized cyclotron waves $(l=-1)$ in the hydrogen plasma at magnetic shell $L=6.6$ with the particle density $N_{e}=N_{c}+N_{h}=11 \mathrm{~cm}^{-3}$.
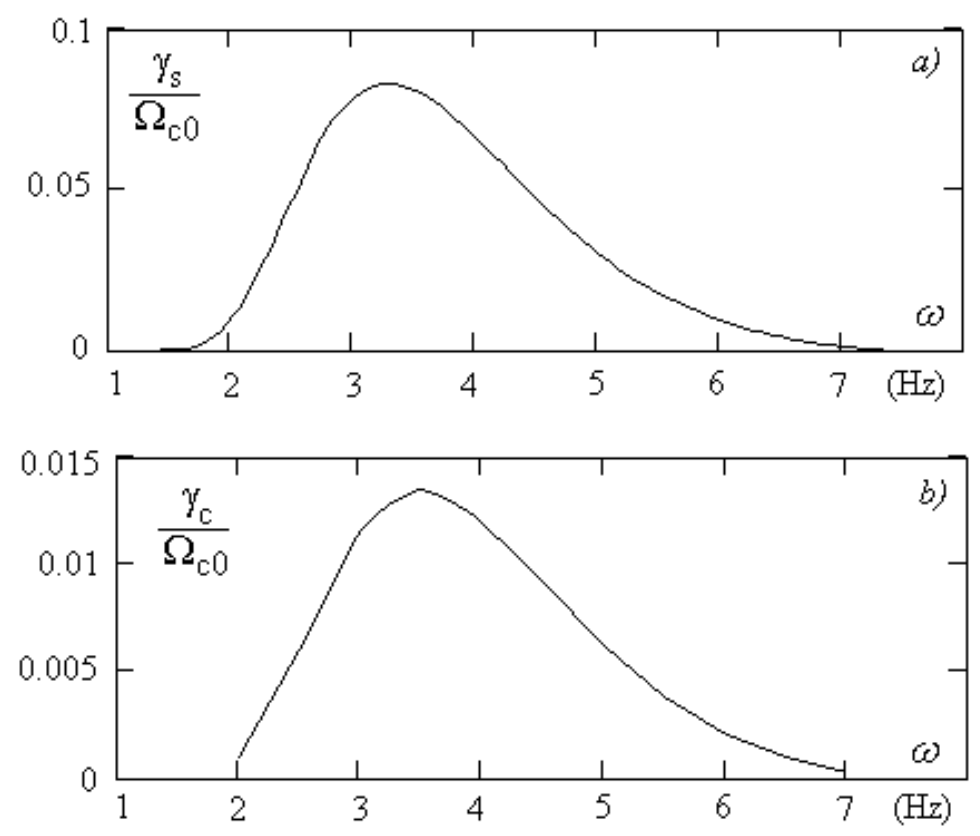

Fig. 3. The growth rates versus $\omega$ for EMIC waves in the hydrogen plasmas confined in the straight magnetic field (a), and in the magnetosphere with circular magnetic field lines (b), under the same macroscopic bulk parameters.

As shown in Figs. 3a and b, the instability of EMIC waves is possible for the both plasma models in the frequency range $\omega<\Omega_{c 0}$. It should be noted that the proton-cyclotron instability is impossible for EMIC waves in the frequency range $\Omega_{c 0}<\omega<\Omega_{c 0} b\left(\theta_{o}\right)$, where $\Omega_{c 0} b\left(\theta_{o}\right)$ is the maximal gyrofrequency of the protons at the given $L$-shell magnetic field line.

As one can see, the dependence of $\gamma_{s}(\omega)$ and $\gamma_{c}(\omega)$ on the wave frequency $\omega$ is similar; however, $\gamma_{c}(\omega) \ll \gamma_{s}(\omega)$ under the same bulk parameters. The ratio $\gamma_{s} / \gamma_{c} \propto 4 \div 10$ versus $\omega$, for considered magnetospheric-like plasmas, is present in Fig. 4. This dependence is not linear; the difference is very large (by factor 10) for EMIC waves in the range of $\omega \sim 2 \mathrm{~Hz}$ and is smaller (by factor 4) in the range of the high frequencies $\omega \sim 7 \mathrm{~Hz}$.
The large difference between $\gamma_{s}$ and $\gamma_{c}$ is connected to the fact that the wave-particle interaction in the straight magnetic field plasma is more effective, since the resonant particles move along the uniform magnetic field line with the constant parallel velocity and interact permanently (in time) with the wave, according to the well-known resonance condition $\omega-\Omega_{c 0}=k_{\|} v_{\|}$. As for 2-D axisymmetric magnetospheric plasmas, since $v_{\|} \neq$const for the trapped particles, there is another wave-particle resonance condition involving the particle energy and pitch angle

$\omega-\Omega_{c 0} \bar{b}(\mu)=p \omega_{b}(v, \mu)$,

where $\omega_{b}(v, \mu)$ is the bounce frequency, $\Omega_{c 0} \bar{b}(\mu)$ is the bounce-averaged gyrofrequency, and $p$ is a integer. As a result, the trapped particle bouncing between the reflection 


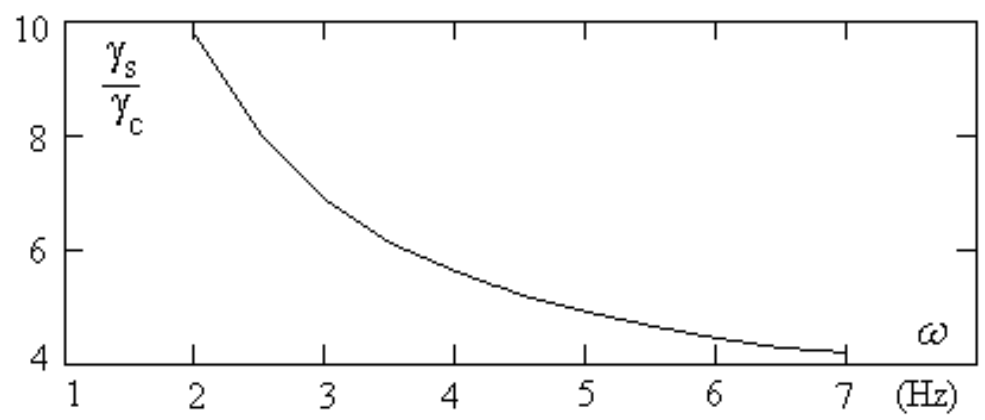

Fig. 4. The ratio $\gamma_{s} / \gamma_{c}$ versus $\omega$ for EMIC waves in the hydrogen 1-D and 2-D plasma models.

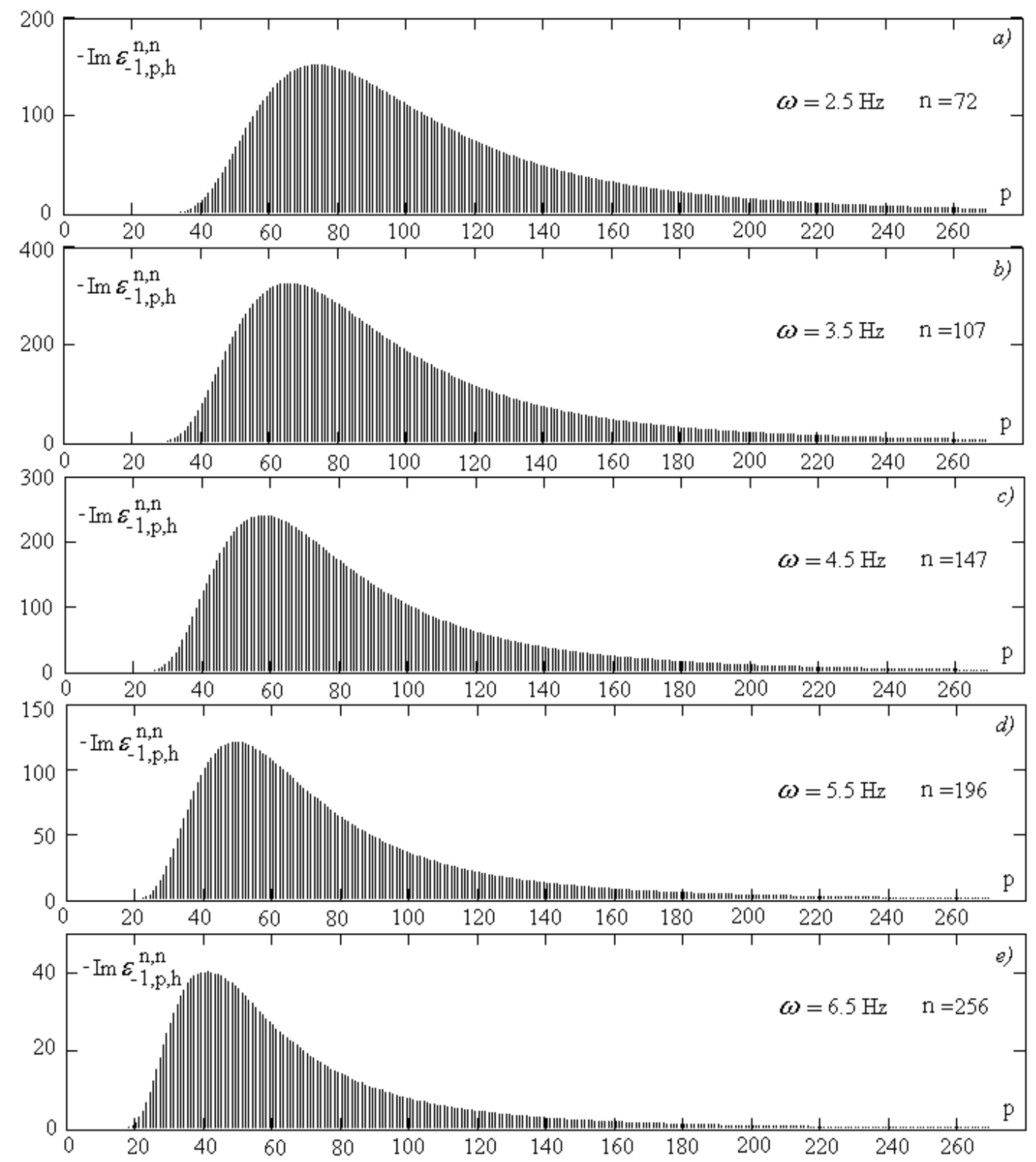

Fig. 5. The separate contributions of the bounce resonant terms to $\operatorname{Im} \varepsilon_{-1, h}^{n, n}$ for the different wave frequencies: (a) $\omega=2.5 \mathrm{~Hz},(\mathbf{b}) \omega=3.5 \mathrm{~Hz}$, (c) $\omega=4.5 \mathrm{~Hz}$, (d) $\omega=5.5 \mathrm{~Hz},(\mathbf{e}) \omega=6.5 \mathrm{~Hz}$. 
points during only part of the bounce-time can interact effectively with the wave.

According to Eqs. (75-77), the PCI growth rate $\gamma_{c}$, as well as the imaginary part of the transverse dielectric permittivity, is calculated by the summation of the contributions of the separate bounce resonant terms to $\operatorname{Im} \varepsilon_{-1, h}^{n, n}$. The typical structure of the bounce resonance terms $\operatorname{Im} \varepsilon_{-1, p, h}^{n, n}$, Eq. (77), is shown in Fig. 5 for the different wave frequencies a) $\omega=2.5 \mathrm{~Hz}$, b) $\omega=3.5 \mathrm{~Hz}$, c) $\omega=4.5 \mathrm{~Hz}$, d) $\omega=5.5 \mathrm{~Hz}$, e) $\omega=6.5 \mathrm{~Hz}$. As we see, i) the contribution of the first bounce resonance terms to $\operatorname{Im} \varepsilon_{-1, h}^{n, n}$ is very small; ii) the maximal values of $\operatorname{Im} \varepsilon_{-1, p, h}^{n, n}$ correspond to the bounce resonance numbers $p_{\max } \sim\left[\omega-\Omega_{c 0} \bar{b}\right] / \omega_{b}$. Accordingly, the $p_{\max }$ numbers are shifted to the left for the EMIC waves with a larger frequency.

\section{Conclusions}

In this paper, we have derived the dispersion equations for field-aligned cyclotron waves in an axisymmetric magnetospheric plasmas with dipole, Eq. (34), and circular, Eq. (64), magnetic field lines, assuming that the energetic "ring current" particles have either the bi-Maxwellian or bi-Lorentzian distribution functions in velocity space. Our dispersion equations can be used to analyze the eigenfrequencies and the temporal growth/damping rates of both the left-hand and right-hand circularly polarized cyclotron waves in both the 2-D magnetosphere-like plasmas. The concrete computations are carried out for the left-hand polarized waves in a hydrogen plasma with circular magnetic field lines. As in the case of a uniform plasma confined in the straight magnetic field, the growth/damping rate of the cyclotron waves in a 2-D magnetosphere is defined by the contribution of the resonant particles to the imaginary part of the transverse dielectric permittivity elements.

To evaluate the contribution of the trapped particles to the transverse current density components the Vlasov equation is solved using a standard method of switching to new variables associated with the conservation integrals of particle energy, magnetic moment and the equation of the geomagnetic field lines. The new time-like variable is introduced (instead of the geomagnetic latitude angle) to describe the bounce-periodic motion of the trapped particles along the 2D geomagnetic field, Eq. (15) and Eq. (47); the perturbed electric field and current density components are Fourierdecomposed over the length of the geomagnetic field lines, Eqs. $(24,25)$ and Eqs. $(55,56)$, for magnetospheric plasmas with dipole and circular magnetic field lines, respectively. As a result, we have derived the contribution of the trapped particles to the transverse permittivity elements for waves in a dipole magnetosphere with bi-Maxwellian distribution functions, Eqs. $(27,28)$; for waves in a dipole magnetosphere with bi-Lorentzian distributions, Eq. (38); and for waves in a magnetospheric plasma with circular magnetic field lines and bi-Maxwellian distribution functions, Eqs. $(58,59)$. The new dielectric characteristics are expressed by summation of the bounce-resonant terms, including the double integration in velocity space, the resonant denominators, and the corresponding phase coefficients. Due to 2-D magnetic field nonuniformity, the bounce-resonance conditions for trapped particles in magnetospheric plasmas are different from the ones in the straight magnetic field; the whole spectrum of the electric field is present in the given current density harmonic; the left-hand and right-hand polarized waves are coupled in the general case.

To have some analogy with the linear theory of cyclotron waves in the straight magnetic field, we assumed that the nth harmonic of the electric field gives the main contribution to the n-th harmonic of the current density and that the connection of the left-hand and right-hand waves is small. In this case, the dispersion equations for field aligned cyclotron waves have the simplest forms and are suitable to analyse the instabilities of both the electron-cyclotron and ion-cyclotron waves, accounting for the cyclotron and bounce resonances.

The comparison of the PCI growth rates is carried out for EMIC waves in the hydrogen plasmas with the straight and circular magnetic field lines under the same bulk magnetosphere-like parameters at the geostationary orbit $L=6.6$. It is shown that the PCI growth rate in the 2-D axisymmetric magnetosphere is much smaller than the corresponding value estimated for EMIC waves in the scope of the 1-D plasma model in the uniform magnetic field. This means that the previous studies of the EMIC waves in the inner magnetosphere, based on the plasma models in the straight magnetic field, could, therefore, be seriously in error.

Of course, a similar approach can be used to analyze the dispersion characteristics of the EMIC waves in the 2-D magnetospheric multi-ions plasmas with dipole and circular magnetic field lines, including the protons and heavy ions (such as $\mathrm{He}^{+}$and $\mathrm{O}^{+}$) with the temperature anisotropy.

It should be noted that the plasma model with circular magnetic field lines (being artificial) is simpler than the model of a dipole magnetosphere and, accordingly, has the more advanced mathematics. Nonetheless, the main physical features of the wave processes, including the cyclotron and bounce resonance wave-particle interactions, in the Earth's magnetosphere, can be analyzed qualitatively by using a model of magnetospheric plasmas with circular magnetic field lines.

Acknowledgements. This research was supported by $\mathrm{CNPq}$ of Brazil (Conselho Nacional de Desenvolvimento Científico e Tecnológico), project PCI-LNCC/MCT 382042/04-2.

Topical Editor I. A. Daglis thanks S. P. Gary and A. Anastasiadis for their help in evaluating this paper. 


\section{References}

Abramovitz, M. and Stegun, I. .: Handbook of mathematical functions, Dover Publication, New York, 1972.

Bespalov, P. A. and Trakhtengerts, V. Yu.: Cyclotron instabilities of the Earth radiation belts, Rev. Plasma Phys., vol. 10, edited by: Leontovich, M. A., Consult Bur., New York, 1986.

Bittencourt, J. A: Fundamentals of plasma physics, Pergamon Press, 1986.

Chan, A. A., Xia, M., and Chen, L.: Anisotropic Alfvén-ballooning modes in Earth's magnetosphere, J. Geophys. Res., 99, A9, 17 351-17366, 1994.

Chen, L. and Hasegawa, A.: Kinetic theory of geomagnetic pulsations: 1.Internal excitations by energetic particles, J. Geophys. Res., 96, 1503-1512, 1991.

Cornwall, J. M.: Cyclotron instabilities and electromagnetic emission in the ultra low frequency andvery low frequency ranges, $\mathrm{J}$. Geophys. Res., 70, 61-69, 1965.

Cuperman, S.: Electromagnetic kinetic instabilities in multicomponent space plasmas, Theoretical predictions and computer simulation experiments, Rev. Geophys., 19, 307-343, 1981.

Dasso, S., Gratton, F. T., and Farrugia, C. J.: The role of alpha particles in the emission of plasma waves inside solar ejecta, Brazilian J. Phys., 32, 632-635, 2002.

Dettrick, S., Zheng, L., and Chen, L.: Kinetic theory of geomagnetic pulsations: 4. Hybrid gyrokinetic simulation of driftbounce resonant excitation of shear Alfven waves, J. Geophys. Res., 108, A4, 1150-1162, 2003.

Fried, B. D. and Conte, S. D.: The plasma dispersion function, Academic, San Diego, California, 1961.

Gary, S. P.: Theory of space plasma microinstabilities, Cambridge Univ. Press, New York, 1993.

Gomberoff, L. and Neira, R.: Convective growth rate of ion cyclotron waves in $\mathrm{a} \mathrm{H}^{+}-\mathrm{He}^{+}$and $\mathrm{H}^{+}-\mathrm{He}^{+}-\mathrm{O}^{+}$plasma, J. Geophys. Res., 88f, 2170-2174, 1983.

Grishanov, N. I., de Azevedo, C. A., and de Assis, A. S.: Longitudinal permittivity of magnetospheric plasmas with dipole and circular magnetic field lines, Phys. Plasmas, 5, 4384-4394, 1998.

Grishanov, N. I., Raupp, M. A., Loula, A. F. D., and Pereira Neto, J.: Influence of bounce resonance effects on the cyclotron wave instabilities in a dipole magnetospheric plasma with anisotropic temperature, 12th Int. Congress on Plasma Physics, Nice, France, 25-29 October, E-proceedings of ICPP-2004: http://hal.ccsd. cnrs.fr/docs/00/00/21/69/PDF/icpp2004-P2-001.pdf, 2004.

Horng, J. T.: Cyclotron instabilities in a hydrogen plasma, Chinese J. Phys., 14, 85-89, 1977.

Karpman, V. I., Meerson, B. I., Mikhailovsky, A. B., and Pokhotelov, O. A.: The effects of bounce resonances on wave growth rates in the magnetosphere, Planet Space Sci., 25, 573585, 1977.

Kennel, C. F. and Petschek, H. E.: Limit on stably trapped particle fluxes, J. Geophys. Res., 71, 1-28, 1966.

Khazanov, G. V., Gamayunov, K. V., and Jordanova, V. K.: Selfconsistent model of magnetospheric ring current and electromagnetic ion cyclotron waves: The 2-7 May 1998 storm, J. Geopgys. Res., 108, A12, 1419-1436, 2003.

Klimushkin, D. Y.: Structure of small-scale standing azimuthal Alfvén waves interacting with high-energy particles in the magnetosphere, Plasma Phys. Reports, 24, 1021-1032, 1998.

Kozyra, J. U., Gravens, T. E., Nagy, A. F., Fontheim, E. G., and Ong, R. S. B.: Effects of energetic heavy ions on electromagnetic ion cyclotron wave generation in the plasmapause region, J. Geophys. Res., 89, 2217-2233, 1984.

Sagdeev, R. Z. and Shafranov, V. D.: On the instability of a plasma with na anisotropic distribution of velocities in a magnetic field, Sov. Physics, JETP English Transl., 12, 130-132, 1961.

Southwood, D. J., Dungey, J. W., and Etherington, R. J.: Bounce resonant interactions between pulsations and trapped particles, Planet. Space Sci., 17, 349-361, 1969.

Summers, D. and Thorne, R. M.: The modified plasma dispersion function, Phys. Fluids, B3, 1835-1847, 1991.

Xue, S., Thorne, R. M., and Summers, D.: Parametric study of electromagnetic ion cyclotron instability in the Earth magnetosphere, J. Geophys. Res., 101 A7, 15 467-15 474, 1996. 